\title{
Multiplex Immunohistochemistry Applies to Examination of PD-1/PD-L1 on Immune Cells for Prognosis Prediction in Non-Small Cell Lung Cancer
}

Huiyong Chen

Yue Bei People's Hospital

Hongliang Liao

Yue Bei People's Hospital

Longlong Gong

Genecast Biotechnology Co.,Ltd.

Jingting Liu

Yue Bei People's Hospital

Lin Zhou

Yue Bei People's Hospital

Lin Kang

Yue Bei People's Hospital

Hongbo Zheng

Genecast Biotechnology Co.,Ltd.

Renping Wan ( $\nabla$ wanrenping1251@163.com)

Yue Bei People's Hospital

\section{Research}

Keywords: PD-L1, PD-1, immune cells, non-small cell lung cancer, prognosis

Posted Date: September 27th, 2021

DOl: https://doi.org/10.21203/rs.3.rs-929708/v1

License: (1) (1) This work is licensed under a Creative Commons Attribution 4.0 International License. Read Full License 


\section{Abstract}

Background: Tumor cells expressing programmed cell death 1 (PD-1) and programmed cell death ligand 1 (PD-L1) correlate with a better prognosis of immunotherapy in non-small cell lung cancer (NSCLC) patients. Expression of PD-1 and PD-L1 on immune cells is also concerned by more and more researchers.

Methods: This study included 174 patients with NSCLC, and collected from the month of December in 2012 to April 2019. Formalin-fixed paraffin-embedded (FFPE) samples from NSCLC patients were performed by multiplex immunohistochemistry (IHC) staining using CD8, CD57, CD68, CD163, PD-1 and PD-L1. Marker localization included each type of immune cell subset with PD-1 or PD-L1 was quantified and analyzed.

Results: The present study revealed distribution characteristics of PD-1 and PD-L1 on CD8+ T cells, CD57+ NK cells, CD68+ macrophages and CD163+ M2 macrophages in NSCLC patients using multiplex IHC, which indicated that expression of PD-1 was higher on CD8+ T cells and expression of PD-L1 was higher on CD8+ T cells and CD68+ macrophages. Immune clustering analysis showed that the low immune feature group displayed more survival rate than the high group. The reason was due to higher ratios of CD8/PD-L1 in the low group compared with the high group in the NSCLC cohort. Further, the Kaplan-Meier analysis of survival rate according infiltration of different immune cells also indicated that low CD57+ NK cells and low CD68+ macrophages were associated with a higher survival rate. The similar results were observed in the Kaplan-Meier analysis of expression of PD-1 and PD-L1 on immune cells.

Conclusions: Taken together, we displayed the expression characteristics of PD-1 and PD-L1 on tumorinfiltrating immune cells and revealed that high expression of PD-1 and PD-L1 on immune cells was associated with poor survival rate. The present study provided further evidence to better guide clinical treatment in NSCLC.

\section{Background}

Lung cancer is one of the most common malignant tumors in the world and one of the leading causes of cancer death $[1,2]$. In the past decade, immune checkpoint inhibitors (ICls) for non-small cell lung cancer (NSCLC) have been made significantly progress, especially the programmed cell death 1 (PD-1) and programmed cell death ligand 1 (PD- L1) [3]. PD-1 and PD-L1 antibodies or inhibitors have been approved for patients with advanced NSCLC who do not respond to platinum-based chemotherapy $[4,5]$. Numerous studies indicate that biomarker screening can effectively select patients suitable for immunotherapy [610].

In the tumor microenvironment, interaction between PD-1 and PD-L1 can cause apoptosis of anti-tumor infiltrating immune cells $[11,12]$. Tumor cells expressing PD-L1 can escape the surveillance of the immune system, especially when PD-1 is expressed on immune cells, thereby promoting tumorigenesis and progression [13]. Some studies have observed that PD-1 could be detected on T cells, macrophages 
and natural killer (NK) cells [14-16]. PD-1, as an immunosuppressive receptor, is expressed only on the surface of activated cells in vivo, but not on resting T cells $[13,17]$. Thus, expression of PD-1 may indicate immune cell activity. In addition, several studies have found that expression of PD-1 on CD8 + cells could be used to predict the effectiveness of ICI treatment in patients with NSCLC [18-20]. The choice of immunotherapy is mainly based on the expression level of PD-L1 in tumor cells in NSCLC, which is closely related to the prognosis [21-23]. Recent studies indicated that expression of PD-L1 has been found not only on tumor cells, but also on immune cells, including cytotoxic T cells, NK cells, macrophages and B cells [10,24-26]. Thus, expression of PD-L1 on immune cells, as a potential predictive biomarker, is concerned by more and more researchers [27, 28].

In the present study, we aimed to investigate distribution characteristics of PD-1 and PD-L1 on immune cells including CD8 + T cells, CD57 + NK cells, CD68 + macrophages and CD163 + M2 macrophages in NSCLC patients using multiplex immunohistochemistry (IHC) and explore the predictive role of PD-1 or PD-L1 expression on different immune cells for prognosis in NSCLC.

\section{Materials And Methods}

\section{Patients and clinical data}

This study included 174 patients with NSCLC, and collected from the month of December in 2012 to April 2019. This study was performed in accordance with the Declaration of Helsinki. And it was approved by the Internal Review and the Ethics Boards of the Yuebei People's Hospital of Shaoguan. Most of patients underwent chemotherapy or targeted therapy or chemotherapy combined with targeted therapy. The clinical and pathological data, including age, gender, smoking history, pathologic stage and tumor histology, was collected for analysis after patient consent (Table 1). 
Table 1

The patient characteristics in the NSCLC cohort.

\begin{tabular}{|c|c|c|c|c|}
\hline Characteristic & \multicolumn{2}{|c|}{$\operatorname{ADC}(n=138)$} & \multicolumn{2}{|c|}{$\operatorname{SQCC}(n=36)$} \\
\hline \multicolumn{5}{|l|}{ Age(years) } \\
\hline Median & \multicolumn{2}{|l|}{61} & \multicolumn{2}{|l|}{67} \\
\hline Range & \multicolumn{2}{|l|}{$29-82$} & \multicolumn{2}{|c|}{$50-84$} \\
\hline \multicolumn{5}{|l|}{ Sex } \\
\hline Male & 85 & $62 \%$ & 34 & $94 \%$ \\
\hline Female & 53 & $38 \%$ & 2 & $6 \%$ \\
\hline \multicolumn{5}{|l|}{ Smoking status } \\
\hline Never smoker & 74 & $54 \%$ & 7 & $20 \%$ \\
\hline Smoker & 60 & $43 \%$ & 26 & $72 \%$ \\
\hline *Missing & 4 & $3 \%$ & 3 & $8 \%$ \\
\hline \multicolumn{5}{|l|}{ Pathologic stage } \\
\hline$\square$ & 15 & $11 \%$ & 1 & $3 \%$ \\
\hline$\square$ & 11 & $8 \%$ & 1 & $3 \%$ \\
\hline$\square$ & 51 & $37 \%$ & 12 & $33 \%$ \\
\hline$\square$ & 55 & $40 \%$ & 13 & $36 \%$ \\
\hline *Missing & 6 & $4 \%$ & 9 & $25 \%$ \\
\hline \multicolumn{5}{|l|}{ Therapy } \\
\hline Target & 46 & $33 \%$ & 6 & $17 \%$ \\
\hline Chemotherapy & 40 & $29 \%$ & 17 & $47 \%$ \\
\hline Chemotherapy and Target & 34 & $25 \%$ & 8 & $22 \%$ \\
\hline Other & 7 & $5 \%$ & 4 & $11 \%$ \\
\hline *Missing & 11 & $8 \%$ & 1 & $3 \%$ \\
\hline
\end{tabular}

\section{Multiplex Ihc Staining}

Based on the previous studies, we selected seven biomarkers involved in this study, including CD8 (cytotoxic T cells; Clone SP16; ZA0508; Zsbio), CD57 (natural killer cells; Clone NK-1; ZM-0058; Zsbio;), CD68 (macrophage; Clone KP1; ZM0060; Zsbio), CD163 (M2 macrophage; Clone 10D6; ZM0428; Zsbio), 
PD-1 (programmed cell death-1; Clone UMAB199; ZM0381; Zsbio) and PD-L1 (programmed cell deathLigand 1; Clone E1L3N; CST13684; Cell Signaling Technology). Formalin-fixed paraffin-embedded (FFPE) samples were cut from NSCLC patients, sections of $4 \mu \mathrm{m}$ thickness. Briefly, the slides were stained manually according to the instruction using the Opal seven-color IHC Kit (NEL797B001 KT; PerkinElmer, Massachusetts, USA), containing fluorophores 4',6-diamidino-2-phenylindole (DAPI), Opal 520 (CD163), Opal 650 (CD68), Opal 570 (PDL1), Opal 540 (CD8), Opal 690 (PD1), Opal 620 (CD57), and TSA Coimarin system (NEL703001KT; PerkinElmer, Massachusetts, USA). Every staining round contained a slide of tonsil as a positive control. Stained slides were scanned by the Vectra (Vectra 3.0.5; PerkinElmer, Massachusetts, USA). After scanning, a selected of 10-15 representative images were used to analysis by the inform software (inform 2.3.0; PerkinElmer, Massachusetts, USA). The multispectral images of the tissue were analyzed in two compartments: tumor area (TA) and stroma area (SA). Marker localization included each type of immune cell subset with PD-1 or PD-L1 was quantified and analyzed.

\section{Statistical analysis}

We compared differences between two groups using the Mann-Whitney $U$ test (unpaired, nonparametric, two-tailed). For every feature using X-tile software (version 3.6.1; Yale University School of Medicine, New Haven, CT) defined the optimum cutoff score based on the association with the patients' survival rate. In univariate analysis for different variable values, survival curves were performed using the Kaplan-Meier method and were compared using log rank test. Statistical analysis was generated using GraphPad Prism (version 7.01), all tests $P<0.05$ was considered significant. In multi-factor analysis, we applied random forest trees for analysis (python software).

\section{Results}

\section{Distribution characteristics of PD-1 and PD-L1 on immune cells}

The NSCLC cohort contained 174 cases, including 138 adenocarcinoma (ADC) cases and 36 squamous cell carcinoma (SQCC) cases (Table 1). The age, gender, smoking history pathologic stage and tumor histology were indicated in Table 1 . The pathologic stage of these patients mainly concentrated in stage III and IV and most of patients underwent chemotherapy or/and targeted therapy. We first explored distribution characteristics of PD-1 and PD-L1 in different immune cells in 174 NSCLC cases. As shown in Fig. 1A and 1B, PD-1 or PD-L1 expression was detected on CD8 + T cells, CD57 + NK cells, CD68 + macrophages and CD163 + M2 macrophages and displayed a similar tendency in stroma area (SA) and tumor area (TA). Expression of PD-1 was higher on CD8 + T cells and expression of PD-L1 was higher on $\mathrm{CD} 8+\mathrm{T}$ cells and CD68 + macrophages in SA and TA (Fig. 1A and 1B). Representative multiple IHC images were showed about expression of PD-1 on CD8 + T cells (Fig. 1C) and expression of PD-L1 on CD8 $+T$ cells (Fig. 1D) and CD68 + macrophages (Fig. 1E). These images also indicated high expression of PD-1 and PD-L1 on CD8 + T cells and high expression of PD-L1 on CD68 + macrophages. 


\section{Expression of PD-1/PD-L1 and infiltration of immune cells predicted the prognosis}

We divided 174 NSCLC cases into high immune feature group and low immune feature group according to immune clustering molecules (Fig. 2A). Specifically, the clustering molecules included the positive rate of T cells (CD8 + cells), NK cells (CD57 + cells), macrophages (CD68 + cells), M2 macrophages (CD163 + cells) and of each immune subpopulation expressing PD-1 or PDL-1 in the TA and SA. As shown in Fig. 2A, the high and low groups contained 100 and 74 NSCLC cases, respectively. According to the high and low groups, we found that the low group displayed more survival rate than the high group $(P=$ 0.0081) (Fig. 2B).

Because of high expression of PD-1 and PD-L1 on CD8 + T cells in Fig. 1, we analyzed ratios of CD8/PD-1 and CD8/PD-L1 in the TA and SA between the low and high immune feature groups. The results indicated that the ratio of CD8/PD-1 was not significant difference in the TA and SA between the low and high groups (Fig. 2C and 2D). However, the ratio of CD8/PD-L1 in the low group was significantly increased in TA and SA compared with the high group (Fig. 2E and 2F). These data showed that expression of PD-L1 on CD8 + T cells in the low group was low, which might be one of important reasons to benefit from survival. Random forest tree analysis further showed that five indicators contributed to the most survival analysis were CD68 + PD-L1+, CD 57 + PD-L1+, CD8 + PD-L1+, CD68 + in SA and CD 68 + PD-L1 + in TA (Fig. 2G). Among them, CD68 + PD-L1 + and CD57 + PD-L1 + have been reported in some literatures and could be used as a screening biomarker for lung cancer immunotherapy $[10,25]$. These results indicated that the immune cells expressing PD-L1 could affect the clinical outcome of patients with NSCLC.

\section{Infiltration of different immune cells predicted the prognosis in the NSCLC cases}

We further explored effects of different immune cells on prognosis in the NSCLC cohort using X-tile software to define the optimum cutoff value for CD8+, CD57+, CD68 + and CD163+. The results indicated that low CD57 + NK cells (Fig. 3A) and low CD68 + macrophages (Fig. 3B) in SA and TA showed a higher survival rate compared with the high group (Table S1). But high/low groups of CD $8+T$ cells (Fig. $3 C$ ) and CD163 + M2 macrophages (Fig. 3D) according to the optimum cutoff value did not show significant difference (Table S1). The data suggested that NSCLC patients could benefit from low CD57 + NK cells and CD68 + macrophages.

\section{Expression of PD-1/PD-L1 on immune cells predicted the prognosis in the NSCLC cases}

We next analyzed expression of PD-1 or PD-L1 on immune cells to predict the survival in the NSCLC cohort. According to the optimum cutoff value (Table S2), low expression of PD-1 on CD8 + T cells (Fig. 4A) and CD57 + NK cells (Fig. 4C) in SA was associated with a higher survival rate. And low expression of PD-L1 on CD8 + T cells (Fig. 4B) and CD $57+$ NK cells (Fig. 4D) in SA and TA showed a better survival rate (Table S2). Interestingly, on CD68 + macrophages, low expression of PD-1 or PD-L1 in either SA or TA displayed a beneficial prognosis (Fig. 4E and 4F, Table S2). But on CD163 + M2 macrophages, low expression of PD-1 or PD-L1 in SA was beneficial for survival in NSCLC patients (Fig. 4G and 4H, Table S2). Taken together, we provided further demonstration which PD-1 and PD-L1 
expression on immune cells as predictive biomarkers was applied to prognosis analysis in NSCLC patients using multiple $\mathrm{IHC}$ detection.

\section{Discussion}

In recent years, immunotherapy has made great progress in the treatment of NSCLC, especially the PD-1 and PD-L1 inhibitors. In order to more accurately screen patients who could benefit from immunotherapy, many studies have reported some biomarkers related to the efficacy of immune checkpoint inhibitors [6$9,18,29]$. Among them, PD-L1 expression on tumor cells was the most widely used. However, several studies have found that some patients whose tumor cells without PD-L1 expressing could also achieve durable response from immunotherapy [30,31]. Moreover, PD-L1 has been observed not only to be expressed on tumor cells, but also broadly expressed on immune cells, which have an important role in immunotherapy. In addition to the expression of PD-L1, the tumor microenvironment was also inextricably linked to the efficacy of ICI [32], and the tumor-infiltrating lymphocytes (TILs) could effectively stratify patients with NSCLC $[33,34]$. Therefore, a better understanding of the expression of PD-1 and PD-L1 distribute over different cells and their impact on clinical outcomes, which could provide insights for the treatment of NSCLC patient. To achieve this objective, we first conducted a detailed retrospective study of the tumor tissue of 174 patients with NSCLC. The results indicate that immune cells expressing PD-1 or PD-L1 were able to predict the clinical prognosis of patients with NSCLC.

A large number of mechanistic research data showed that PD-1 was expressed primarily on T cells and PD-L1 was mainly expressed on tumor cells. Combination of PD-1 and PD-L1 results in tumor immune evasion. In the present study, we found that PD-1 and PD-L1 were expressed on T cells, NK cells and macrophages using multiplex IHC detection. The average positive rates of PD-1 expression on CD8 + T cells, CD57 + NK cells, CD $68+$ macrophages and CD163 + M2 macrophages were $14.00 \%, 4.17 \%, 5.96 \%$ and $9.61 \%$ in SA, and $12.27 \%, 2.64 \%, 3.07 \%$ and $4.89 \%$ in TA, respectively. The average positive rates of PD-L1 expression on CD8 + T cells, CD57 + NK cells, CD68 + macrophages and CD163 + M2 macrophages were $13.30 \%, 5.61 \%, 12.23 \%$ and $8.5 \%$ in SA, and $6.86 \%, 2.88 \%, 6.22 \%$ and $2.5 \%$ in TA, respectively. PD-L1 distribution in immune cells was mainly expressed on macrophages and T cells. This type of macrophages (CD68 + PD-L1+) were probably related to the promotion of immune escape and belonged to tumor associated macrophages (TAM) [35]. However, there were few studies about PD-L1 expression on $\mathrm{T}$ cells, which is needed further explore.

According to the positive rates of immune profiling, the NSCLC cohort were divided into a strong immune feature group (high) and a weak immune feature group (low). Interestingly, we observed a better survival rate in patients from the low group, suggesting that tumor microenvironment in the low group may be biased towards the activated state, while the high group was the opposite. The ratio analysis of CD8/PD1 and CD8/PD-L1 in TA and SA revealed that the low group had higher ratio of CD8/PD-1 and CD8/PD-L1, especially the CD8/PD-L1 ratio was significantly different between two groups. Meanwhile, multivariate analysis of random forest trees in two groups indicated that CD68 + PD-L1+, CD57 + PD-L1+, CD8 + PD$\mathrm{L} 1+, \mathrm{CD} 68$ + in SA and CD68 + PD-L1 + in TA had a major effect on survival rate. These results 
demonstrated that more immune cells expressing PD-1 or PD-L1 in the high group cause the suppression of immune cells, leading to decrease of survival rate.

The immune profiling had shown a significant impact on the survival outcome of patients with NSCLC. Effects of the single immune characteristic on the survival were further explored. In the study, 16 immune characteristics (including CD57+, CD68+, CD $8+$ PD-L1+, CD 57 + PD-L1+, CD 68 + PD-1 + and CD 68 + PD-L1 + in TA, and CD 57+, CD68+, CD8 + PD-1+, CD8 + PD-L1+, CD 57 + PD-1+, CD 57 + PD-L1+, CD 68 + PD-1+, CD68 + PD-L1+, CD163 + PD-1 + and CD163 + PD-L1 + in SA) according to the optimum cutoff values could effectively distinguish the difference of survival rate in the NSCLC cohort. Results of the KaplanMeier survival analysis of these categories were consistent with conclusion of the immune feature analysis, which the more immune cell infiltration exhibited the worse of survival rate in the NSCLC cases. However, several studies discovered that increased NK cells (CD57+) and macrophages (CD68+) infiltration in lung cancer were associated with good clinical outcome [36, 37]. Expression of PD-1/PD-L1 on immune cells was a key factor causing the different results. Owing to the retrospective NSCLC cohort untreated with immunotherapy, association of PD-1/PD-L1 expression on immune cells and outcomes of immunotherapy was not discussed. Taken together, these demonstrations indicated that immune cells expressing PD-1 or PD-L1 could predict the clinical outcome of patients with NSCLC.

\section{Conclusions}

In summary, our results indicated that expression characteristics of PD-1 and PD-L1 on immune cells could predict the clinical prognosis of patients with NSCLC. The NSCLC patients with more immune cells expressing PD-1 or PD-L1 were associated with poor survival rate. The present study provided further evidence to better guide clinical treatment in NSCLC.

\section{Abbreviations}

immune checkpoint inhibitors

ICls

non-small cell lung cancer

NSCLC

programmed cell death 1

PD-1

programmed cell death ligand 1

PD- L1

natural killer cells

NK cells

immunohistochemistry

IHC

formalin-fixed paraffin-embedded

FFPE 
tumor area

TA

stroma area

SA

adenocarcinoma

$A D C$

squamous cell carcinoma

SQCC

tumor-infiltrating lymphocytes

TILS

tumor associated macrophages

TAM

\section{Declarations}

\section{Ethics approval and consent to participate}

The study was approved by the Ethics Committee of The Yuebei People's Hospital of Shaoguan. Signed written informed consents were obtained from the patients and/or guardians.

\section{Consent for publication}

Not applicable.

\section{Availability of data and materials}

The datasets used and/or analysed during the current study are available from the corresponding author on reasonable request.

\section{Competing interests}

Two of the authors (LG and HZ) affiliated with Genecast Biotechnology Co., Ltd performed multiplex IHC test and analysis. The other authors declare that they have no competing interests.

\section{Funding}

None.

\section{Authors' contributions}

RW and $\mathrm{HC}$ conceived and designed the research. RW, HC, HL, GL, JT and LZ performed the experiments. $\mathrm{HL}, \mathrm{GL}$ and $\mathrm{HZ}$ analyzed the experiment data. $\mathrm{HC}$ and $\mathrm{LG}$ wrote the manuscript. RW and $\mathrm{HC}$ revised the manuscript. 


\section{Acknowledgments}

The authors wish to thank the patients and their families.

\section{References}

1. Siegel RL, Miller KD, Jemal A. Cancer statistics, 2019. CA Cancer J Clin. 2019;69:7-34.

2. Chen W, Zheng R, Baade PD, Zhang S, Zeng H, Bray F, Jemal A, Yu XQ, He J. Cancer statistics in China, 2015. CA Cancer J Clin. 2016;66:115-32.

3. Heigener DF, Reck M. Advanced non-small cell lung cancer: the role of PD-L1 inhibitors. J Thorac Dis. 2018;10:1468-73.

4. Reck M, Rodriguez-Abreu D, Robinson AG, Hui R, Csoszi T, Fulop A, Gottfried M, Peled N, Tafreshi A, Cuffe S, et al. Pembrolizumab versus Chemotherapy for PD-L1-Positive Non-Small-Cell Lung Cancer. N Engl J Med. 2016;375:1823-33.

5. Rittmeyer A, Barlesi F, Waterkamp D, Park K, Ciardiello F, von Pawel J, Gadgeel SM, Hida T, Kowalski $\mathrm{DM}$, Dols MC, et al. Atezolizumab versus docetaxel in patients with previously treated non-small-cell lung cancer (OAK): a phase 3 , open-label, multicentre randomised controlled trial. Lancet. 2017;389:255-65.

6. Shukuya T, Carbone DP. Predictive Markers for the Efficacy of Anti-PD-1/PD-L1 Antibodies in Lung Cancer. J Thorac Oncol. 2016;11:976-88.

7. Rizvi NA, Hellmann MD, Snyder A, Kvistborg P, Makarov V, Havel JJ, Lee W, Yuan J, Wong P, Ho TS, et al. Cancer immunology. Mutational landscape determines sensitivity to PD-1 blockade in non-small cell lung cancer. Science. 2015;348:124-8.

8. Dong ZY, Wu SP, Liao RQ, Huang SM, Wu YL. Potential biomarker for checkpoint blockade immunotherapy and treatment strategy. Tumour Biol. 2016;37:4251-61.

9. Luksza M, Riaz N, Makarov V, Balachandran VP, Hellmann MD, Solovyov A, Rizvi NA, Merghoub T, Levine AJ, Chan TA, et al. A neoantigen fitness model predicts tumour response to checkpoint blockade immunotherapy. Nature. 2017;551:517-20.

10. Liu Y, Zugazagoitia J, Ahmed FS, Henick BS, Gettinger SN, Herbst RS, Schalper KA, Rimm DL. Immune Cell PD-L1 Colocalizes with Macrophages and Is Associated with Outcome in PD-1 Pathway Blockade Therapy. Clin Cancer Res 2019.

11. Zhu X, Lang J. Soluble PD-1 and PD-L1: predictive and prognostic significance in cancer. Oncotarget. 2017;8:97671-82.

12. Zhang J, Gao J, Li Y, Nie J, Dai L, Hu W, Chen X, Han J, Ma X, Tian G, et al. Circulating PD-L1 in NSCLC patients and the correlation between the level of PD-L1 expression and the clinical characteristics. Thorac Cancer. 2015;6:534-8.

13. Xu-Monette ZY, Zhang M, Li J, Young KH: PD-1/PD-L1 Blockade: Have We Found the Key to Unleash the Antitumor Immune Response? Front Immunol 2017, 8:1597. 
14. Gordon SR, Maute RL, Dulken BW, Hutter G, George BM, McCracken MN, Gupta R, Tsai JM, Sinha R, Corey D, et al. PD-1 expression by tumour-associated macrophages inhibits phagocytosis and tumour immunity. Nature. 2017;545:495-9.

15. Wang F, Li B, Wei Y, Zhao Y, Wang L, Zhang P, Yang J, He W, Chen H, Jiao Z, Li Y. Tumor-derived exosomes induce PD1(+) macrophage population in human gastric cancer that promotes disease progression. Oncogenesis. 2018;7:41.

16. Liu Y, Cheng Y, Xu Y, Wang Z, Du X, Li C, Peng J, Gao L, Liang X, Ma C. Increased expression of programmed cell death protein 1 on NK cells inhibits NK-cell-mediated anti-tumor function and indicates poor prognosis in digestive cancers. Oncogene. 2017;36:6143-53.

17. Jiang Y, Li Y, Zhu B. T-cell exhaustion in the tumor microenvironment. Cell Death Dis. 2015;6:e1792.

18. Mazzaschi G, Madeddu D, Falco A, Bocchialini G, Goldoni M, Sogni F, Armani G, Lagrasta CA, Lorusso B, Mangiaracina C, et al. Low PD-1 Expression in Cytotoxic CD8(+) Tumor-Infiltrating Lymphocytes Confers an Immune-Privileged Tissue Microenvironment in NSCLC with a Prognostic and Predictive Value. Clin Cancer Res. 2018;24:407-19.

19. Thommen DS, Koelzer VH, Herzig P, Roller A, Trefny M, Dimeloe S, Kiialainen A, Hanhart J, Schill C, Hess $C$, et al. A transcriptionally and functionally distinct PD-1(+) CD8(+) T cell pool with predictive potential in non-small-cell lung cancer treated with PD-1 blockade. Nat Med. 2018;24:994-1004.

20. Lee JS, Ruppin E. Multiomics Prediction of Response Rates to Therapies to Inhibit Programmed Cell Death 1 and Programmed Cell Death 1 Ligand 1. JAMA Oncol 2019.

21. Aguiar PN Jr, De Mello RA, Hall P, Tadokoro H, Lima Lopes G. PD-L1 expression as a predictive biomarker in advanced non-small-cell lung cancer: updated survival data. Immunotherapy. 2017;9:499-506.

22. Garon EB, Hellmann MD, Rizvi NA, Carcereny E, Leighl NB, Ahn MJ, Eder JP, Balmanoukian AS, Aggarwal C, Horn L, et al. Five-Year Overall Survival for Patients With Advanced NonSmall-Cell Lung Cancer Treated With Pembrolizumab: Results From the Phase I KEYNOTE-001 Study. J Clin Oncol. 2019;37:2518-27.

23. Mok TSK, Wu YL, Kudaba I, Kowalski DM, Cho BC, Turna HZ, Castro G Jr, Srimuninnimit V, Laktionov KK, Bondarenko I, et al. Pembrolizumab versus chemotherapy for previously untreated, PD-L1expressing, locally advanced or metastatic non-small-cell lung cancer (KEYNOTE-042): a randomised, open-label, controlled, phase 3 trial. Lancet. 2019;393:1819-30.

24. Mitchell KG, Negrao MV, Parra ER, Li J, Zhang J, Dejima H, Vaporciyan AA, Swisher SG, Weissferdt A, Antonoff MB, et al. Lymphovascular Invasion Is Associated With Mutational Burden and PD-L1 in Resected Lung Cancer. Ann Thorac Surg. 2020;109:358-66.

25. Dong W, Wu X, Ma S, Wang Y, Nalin AP, Zhu Z, Zhang J, Benson DM, He K, Caligiuri MA, Yu J. The Mechanism of Anti-PD-L1 Antibody Efficacy against PD-L1-Negative Tumors Identifies NK Cells Expressing PD-L1 as a Cytolytic Effector. Cancer Discov. 2019;9:1422-37.

26. Sun X, Zhang T, Li M, Yin L, Xue J: Immunosuppressive B cells expressing PD-1/PD-L1 in solid tumors: A mini review. QJM 2019. 
27. Kowanetz M, Zou W, Gettinger SN, Koeppen H, Kockx M, Schmid P, Kadel EE 3rd, Wistuba I, Chaft J, Rizvi NA, et al. Differential regulation of PD-L1 expression by immune and tumor cells in NSCLC and the response to treatment with atezolizumab (anti-PD-L1). Proc Natl Acad Sci U S A. 2018;115:E10119-26.

28. Bocanegra A, Fernandez-Hinojal G, Zuazo-Ibarra M, Arasanz H, Garcia-Granda MJ, Hernandez C, Ibañez M, Hernandez-Marin B, Martinez-Aguillo M, Lecumberri MJ, et al: PD-L1 Expression in Systemic Immune Cell Populations as a Potential Predictive Biomarker of Responses to PD-L1/PD-1
Blockade Therapy in Lung Cancer. Int J Mol Sci 2019, 20.

29. Le DT, Durham JN, Smith KN, Wang H, Bartlett BR, Aulakh LK, Lu S, Kemberling H, Wilt C, Luber BS, et al. Mismatch repair deficiency predicts response of solid tumors to PD-1 blockade. Science. 2017;357:409-13.

30. Brahmer J, Reckamp KL, Baas P, Crino L, Eberhardt WE, Poddubskaya E, Antonia S, Pluzanski A, Vokes EE, Holgado E, et al. Nivolumab versus Docetaxel in Advanced Squamous-Cell Non-Small-Cell Lung Cancer. N Engl J Med. 2015;373:123-35.

31. Robert C, Long GV, Brady B, Dutriaux C, Maio M, Mortier L, Hassel JC, Rutkowski P, McNeil C, KalinkaWarzocha $\mathrm{E}$, et al. Nivolumab in previously untreated melanoma without BRAF mutation. N Engl $\mathrm{J}$ Med. 2015;372:320-30.

32. Haratani K, Hayashi H, Tanaka T, Kaneda H, Togashi Y, Sakai K, Hayashi K, Tomida S, Chiba Y, Yonesaka $\mathrm{K}$, et al. Tumor immune microenvironment and nivolumab efficacy in EGFR mutationpositive non-small-cell lung cancer based on T790M status after disease progression during EGFRTKI treatment. Ann Oncol. 2017;28:1532-9.

33. Kim TK, Herbst RS, Chen L. Defining and Understanding Adaptive Resistance in Cancer Immunotherapy. Trends Immunol. 2018;39:624-31.

34. Chen L, Cao MF, Zhang X, Dang WQ, Xiao JF, Liu Q, Tan YH, Tan YY, Xu YY, Xu SL, et al. The landscape of immune microenvironment in lung adenocarcinoma and squamous cell carcinoma based on PD-L1 expression and tumor-infiltrating lymphocytes. Cancer Med. 2019;8:7207-18.

35. Pollari M, Bruck O, Pellinen T, Vahamurto P, Karjalainen-Lindsberg ML, Mannisto S, Kallioniemi O, Kellokumpu-Lehtinen PL, Mustjoki S, Leivonen SK, Leppa S. PD-L1(+) tumor-associated macrophages and PD-1(+) tumor-infiltrating lymphocytes predict survival in primary testicular lymphoma. Haematologica. 2018;103:1908-14.

36. Villegas FR, Coca S, Villarrubia VG, Jimenez R, Chillon MJ, Jareno J, Zuil M, Callol L. Prognostic significance of tumor infiltrating natural killer cells subset CD57 in patients with squamous cell lung cancer. Lung Cancer. 2002;35:23-8.

37. Parra ER, Behrens C, Rodriguez-Canales J, Lin H, Mino B, Blando J, Zhang J, Gibbons DL, Heymach JV, Sepesi B, et al. Image Analysis-based Assessment of PD-L1 and Tumor-Associated Immune Cells Density Supports Distinct Intratumoral Microenvironment Groups in Non-small Cell Lung Carcinoma Patients. Clin Cancer Res. 2016;22:6278-89. 


\section{Figures}
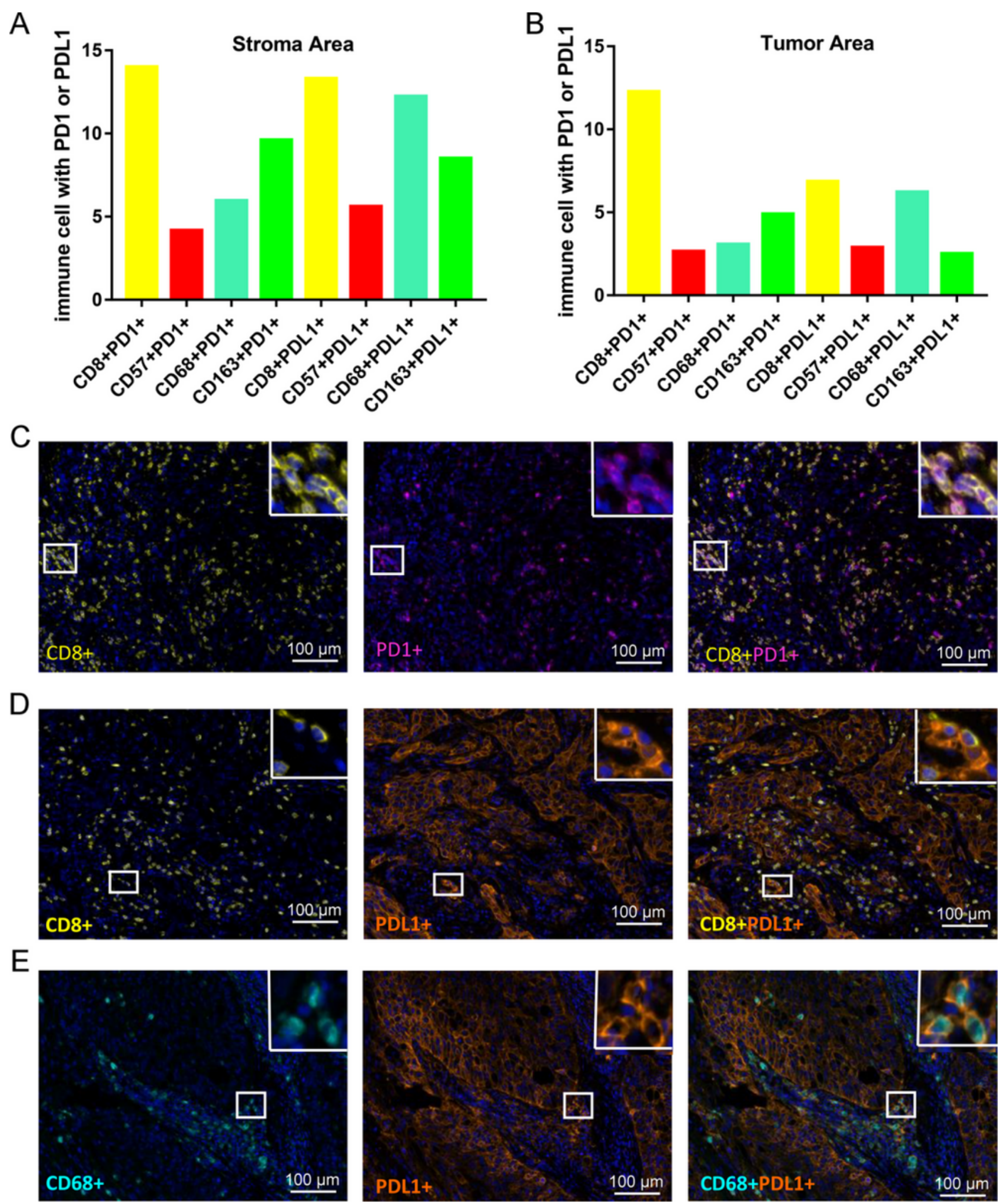

Figure 1

Distribution characteristics of PD-1/PD-L1 on immune cells in the NSCLC cases. (A and B) PD-1 or PD-L1 expression was detected on CD8+ T cells, CD57+ NK cells, CD68+ macrophages and CD163+ M2 macrophages in stroma area (SA) (A) and tumor area (TA) (B). (C-E) Representative multiple IHC images 
of PD-1 expression on CD8+ T cells (C) and PD-L1 expression on CD8+ T cells (D) and CD68+ macrophages (E). Scale bar, $100 \mu \mathrm{m}$.
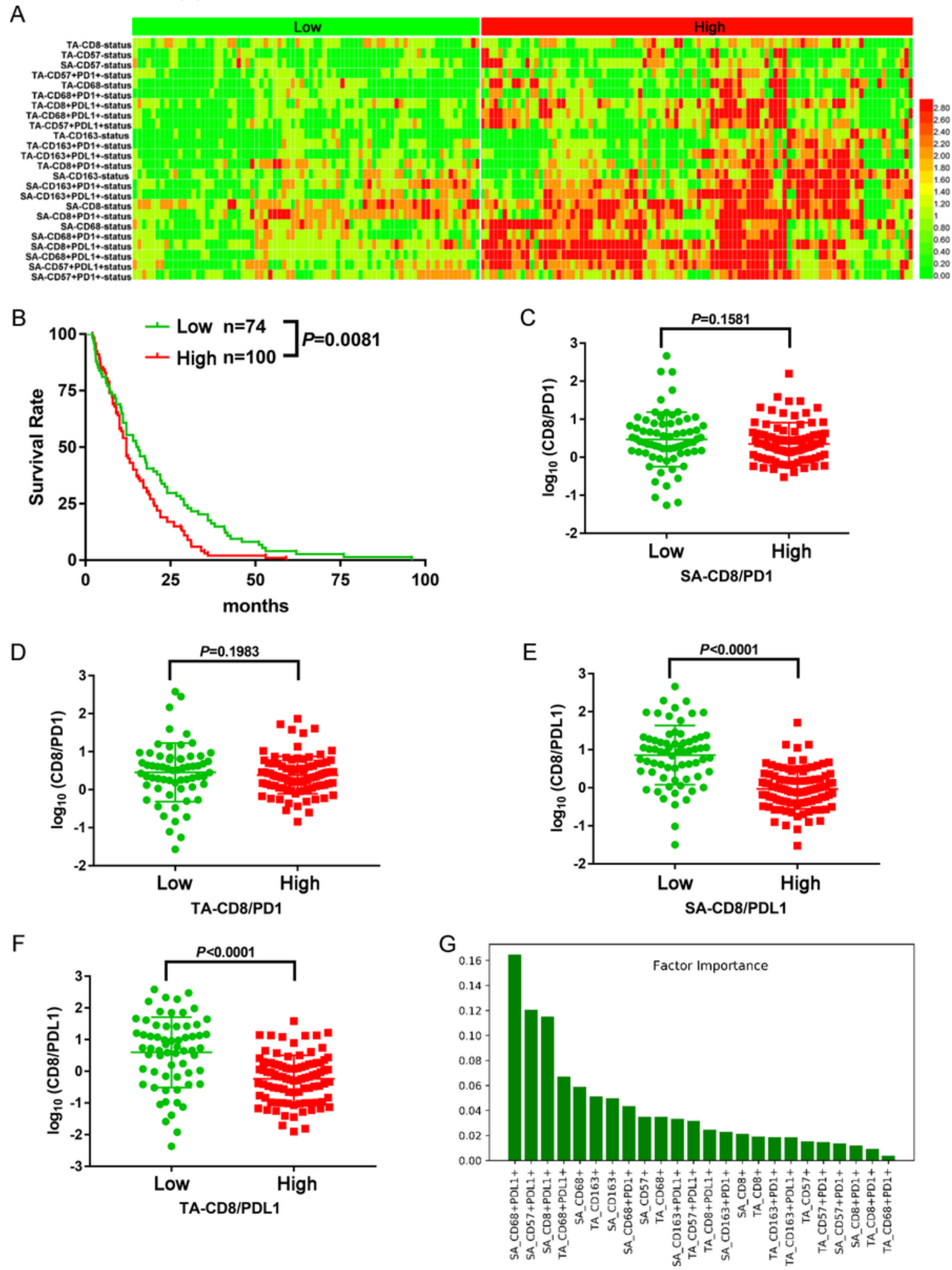

Figure 2

Expression of PD-1/PD-L1 and infiltration of immune cells predicted the prognosis. (A) The NSCLC cohort was divided into low and high immune feature groups according to the positive rate of CD8+ cells, CD57+ cells, CD68+ cells, CD163+ cells and of each immune subpopulation expressing PD-1 or PD-L1 in the TA 
and SA. nlow $=74$; nhigh $=100$. (B) The Kaplan-Meier survival analysis between the high/low groups in the NSCLC cohort. nlow $=74$; nhigh $=100$; Statistics based on the log-rank (Mantel-cox) test; $P=0.0081$. (C and D) The ratio analysis of CD8/PD-1 in SA (C) and TA (D) between low and high groups. Statistics based on the log-rank test. (E and F) The ratio analysis of CD8/PD-L1 in SA (E) or TA (F). Statistics based on the log-rank test. $(G)$ Random forest tree analysis between the high/low groups. $P<0.05$ was considered statistically significant.
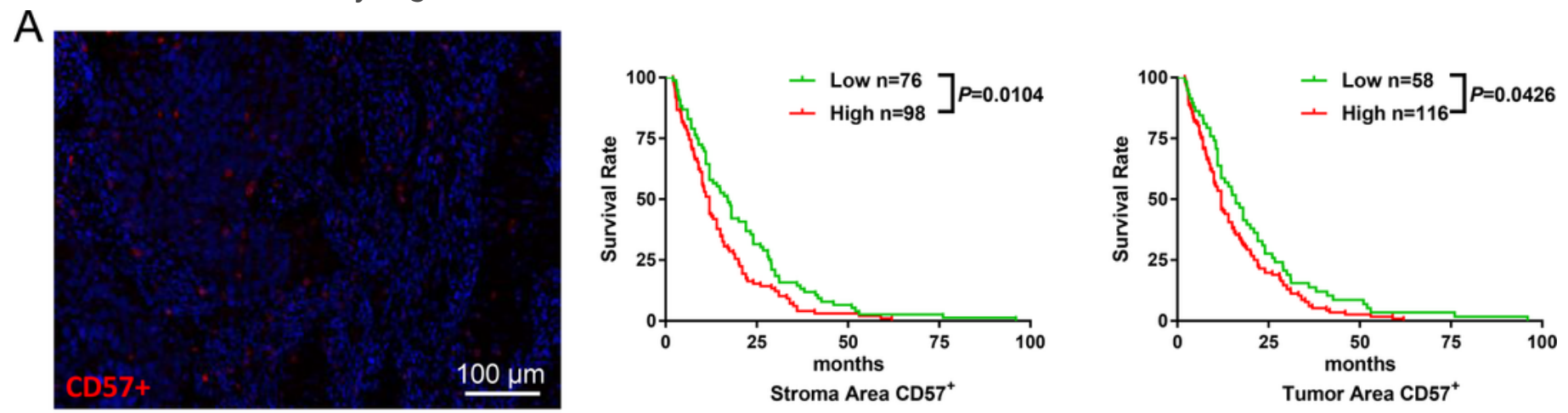

B
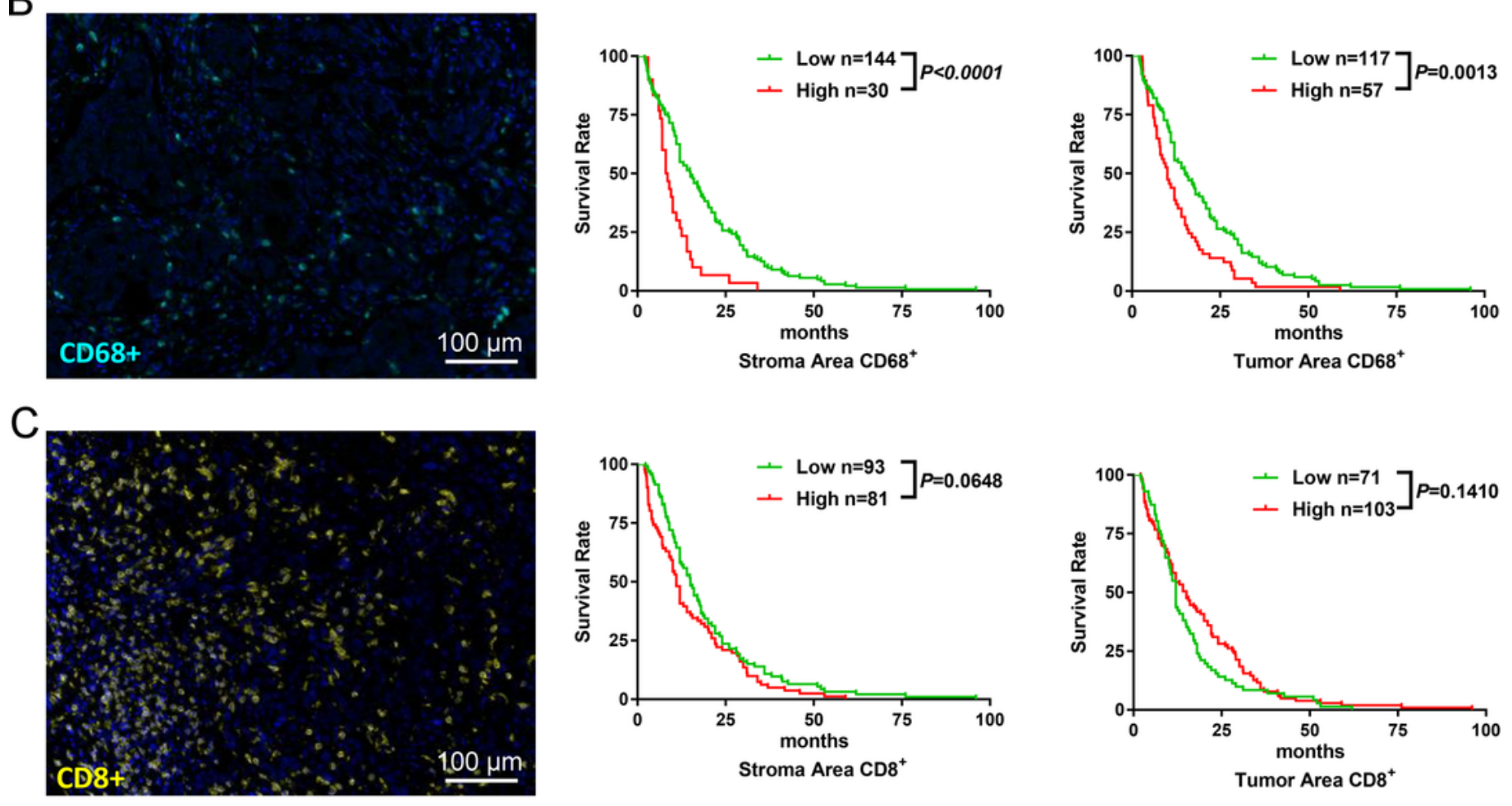

D
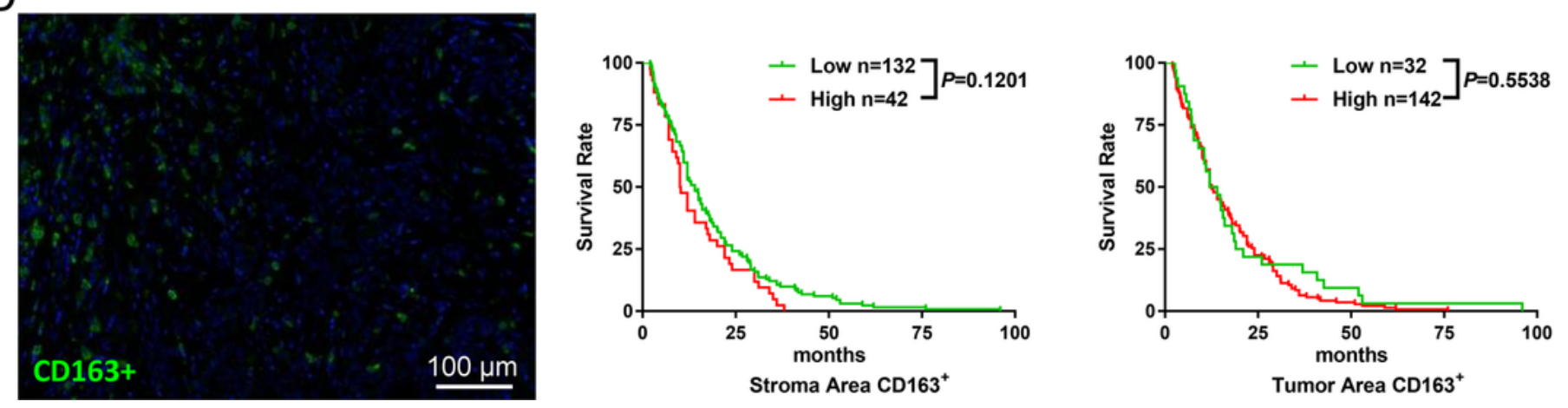

Figure 3 
Infiltration of different immune cells predicted the prognosis in the NSCLC cohort. (A-D) The Kaplan-Meier survival analysis according to infiltration of CD57+ NK cells (A) and CD68+ macrophages (B) CD8+ T cells (C) and CD163+ M2 macrophages (D) using the optimum cutoff value for CD8+, CD57+, CD68+ and CD163+. Scale bar, $100 \mu \mathrm{m}$. Statistics based on the log-rank (Mantel-cox) test; $P<0.05$ was considered statistically significant.

A

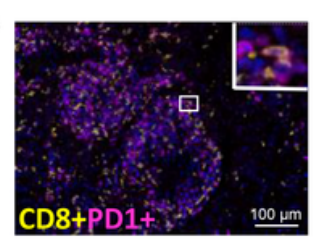

C

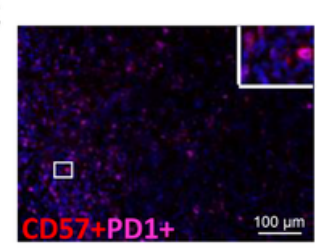

$\mathrm{E}$

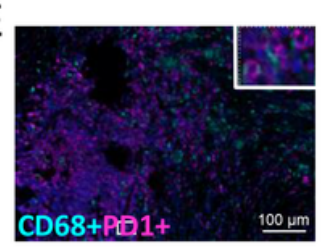

G

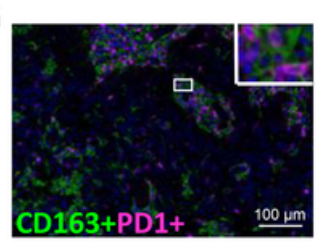

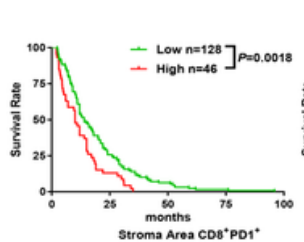
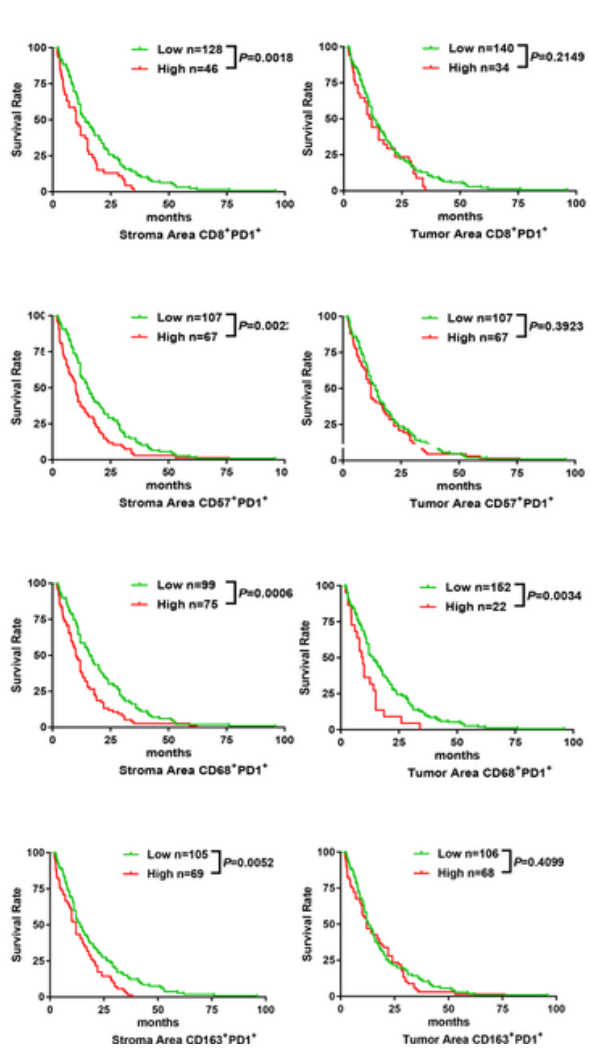
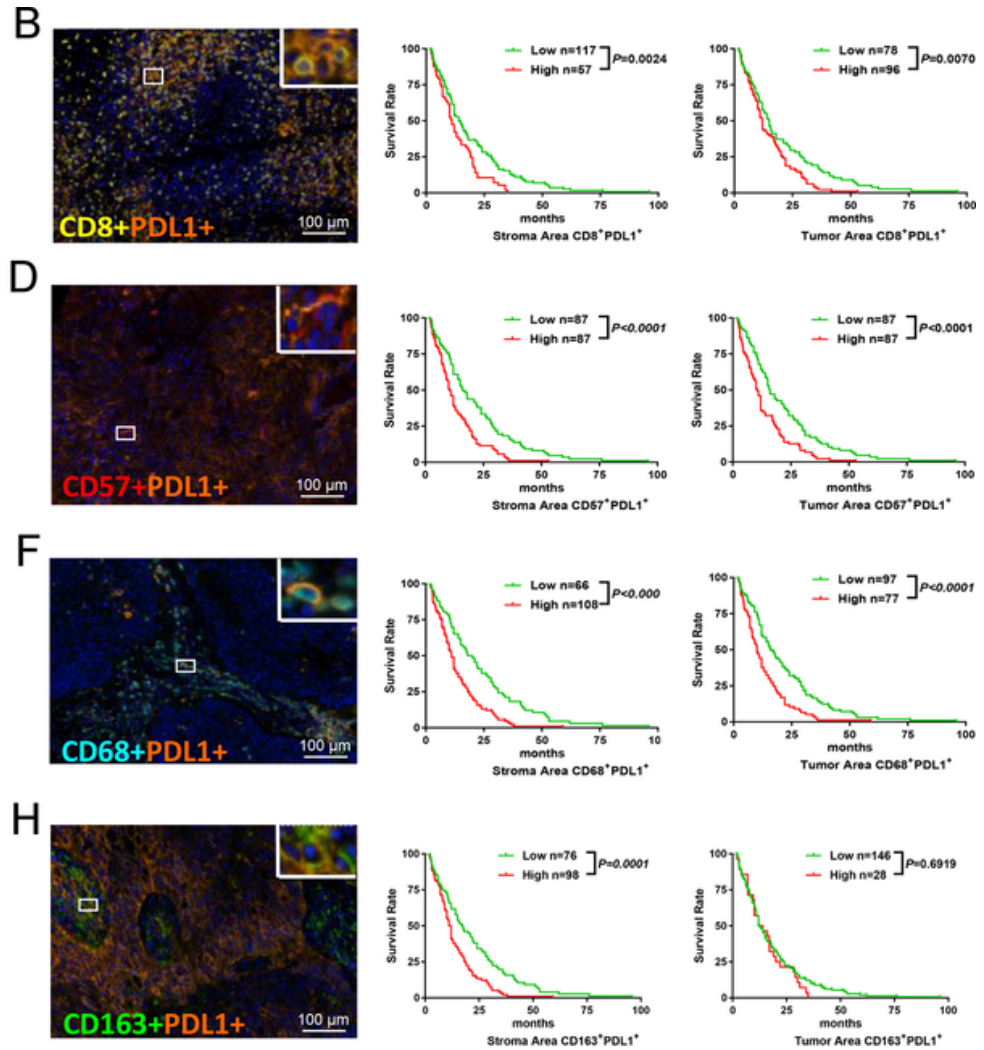

Figure 4

Expression of PD-1/PD-L1 on immune cells predicted the prognosis in the NSCLC cohort. Representative multiple IHC images and the Kaplan-Meier survival analysis using the optimum cutoff value of PD-1 or PD-L1 expression in CD8+ cells ( $A$ and B), CD57+ cells ( $C$ and D), CD68+ cells ( $E$ and F) and CD163+ cells ( $G$ and $H$ ). Scale bar, $100 \mu \mathrm{m}$. Statistics based on the log-rank (Mantel-cox) test; $P<0.05$ was considered statistically significant.

\section{Supplementary Files}

This is a list of supplementary files associated with this preprint. Click to download.

- SupplementaryMaterial.docx 